\title{
LIEUX DE FRANCIS WOLFF
}

\author{
Jean-Michel SALANSKIS
}

Université Paris-Nanterre

Avec Francis Wolff, j'ai d'abord eu le sentiment d'une affinité, qui au fil des années a pris pour moi le visage d'une complicité. Pour le dire simplement, je le percevais comme un des rares qui fût réellement tolérant à l'égard des nombreuses manières de faire de la philosophie : j'entends ici tolérant non seulement au sens de celui qui n'a pas le projet de moquer ou calomnier les autres - ceux qui écrivent autrement, qui ont une autre bibliothèque de référence - mais au sens de celui qui entend et comprend le génie de plusieurs démarches, extrêmement différentes, et même s'il ne leur adhère pas. À vrai dire et par parenthèse, il est triste de l'observer, fort rares sont de nos jours dans la communauté philosophique ceux à qui il est facile de concéder que des gens travaillant tout à fait en dehors d'eux, sans intersection avec aucune de leurs écuries, sont excellents.

Cette faculté pluraliste de Francis Wolff, elle s'apercevait d'emblée si l'on voulait bien considérer qu'il était à la fois un spécialiste reconnu de la philosophie ancienne, et un lecteur engagé de la philosophie contemporaine, réagissant à ses propositions et s'efforçant d'y faire valoir son message propre. Mais à l'intérieur même de cette dernière facette, un second double visage le recommandait à mon estime : il était à la fois quelqu'un de soucieux de la philosophie comme telle, assumant ce que l'on pourrait appeler "philosophie de la philosophie", se refusant à dissoudre dans d'autres compétences l'acquis traditionnel, et quelqu'un d'ouvert sur les cas, régimes ou objets appelant de notre part un philosopher dédié (quelqu'un qui pouvait écrire sur la corrida, par exemple).

Une telle figure, Francis Wolff n'a pu la tenir qu'en s'en faisant le militant. À cet égard, je dois témoigner ici de ce que je lis aujourd'hui, rétrospectivement, comme une incompréhension, qui m'a quelque temps retenu en deçà d'une meilleure proximité avec sa sensibilité. Un des «actes » portant la signature de Francis Wolff, dans notre petit monde, est le séminaire qu'il a tenu, à Nanterre puis à l'ENS Ulm, dans le but de donner la parole à de réelles propositions philosophiques, soutenant quelque chose à propos de 
quelque chose, au lieu de se cantonner à ruminer les alternatives nôtres dans la compréhension et la réception des grands messages reçus. « Positions et arguments », on le sait, fut à l'origine le nom de ce séminaire, qui a perduré par la suite au sein de la série Les lundis de la philosophie. Dans l'argumentaire qui définissait le séminaire, à Nanterre au tout début du nouveau siècle, figurait selon mon souvenir la demande d'interventions qui traitassent des realia plutôt que des dicta. Pour sûr, le texte s'empressait de préciser que l'on ne se fixait pas, en l'espèce, une notion bornée des realia, ce qui permettait, on le devinait, de récupérer des intentions tournées vers du social, du symbolique et du textuel. Néanmoins, je me sentais rebuté par cette formule, qui me semblait ignorer la différence qu'il y avait entre proposer véritablement une conceptualisation nouvelle, et proposer un morceau de discours théorique prétendant couvrir une classe de data. Comme je ressentais la philosophie analytique contemporaine comme embarquée (le plus souvent, ou encore officiellement à tout le moins) dans l'idée que la philosophie devrait se replier sur le second exercice, je voyais le projet de Francis Wolff, malgré le démenti que me donnait régulièrement le choix de ses intervenants, comme faisant pencher la barque du côté analytique.

Avec le recul, je pense aujourd'hui que ce que voulait véritablement Wolff, c'est que les philosophes formés dans le style interprétatif et de la mise en abîme (c'est-à-dire, tous, la donne institutionnelle étant ce que l'on sait) fissent l'effort de préparer des conférences ayant un tour thétique. Il voulait les convaincre d'entrer dans le jeu de l'assertion, de la prévention des objections, de la machinerie argumentative, afin de les aguerrir et de les rendre potentiellement audibles par ceux qui, depuis des décennies, marchaient au paper corrigeant sur un point archi-précis une thèse totalement explicite. Une intention que j'ai moi-même reprise à mon compte, bien que formulée de façon un peu différente, dans le projet sur trois ans «Philosophie française et philosophie analytique au $\mathrm{XX}^{\mathrm{e}}$ siècle » que nous animons avec Elie During, Dan Smith, Arkady Plotnitzky et Sandor Goodhart. Donc je me convaincs que ma lecture était mauvaise, et que nous étions beaucoup plus proches que je ne le supposais alors.

$\mathrm{Au}$ chapitre de la peinture générale préalable, je voudrais mentionner deux traits que j'ai observés de longue date chez Francis Wolff, et qui forçaient ma sympathie $a$ priori, avant que je comprisse mieux de quel ordre était sa pensée : sa volonté d'apporter une clarté synthétique sur ce qui était en débat à un moment donné dans un groupe d'une part, sa capacité à raisonner sur les dossiers, c'est-à-dire les individus, dans le contexte 
des commissions de spécialistes aujourd'hui rebaptisées comités de sélection, sans allergie, sans mépris, à rechercher des raisons positives de prendre plutôt $\mathrm{A}$ que $\mathrm{B}, \mathrm{C}$, et D (c'est-à-dire de distribuer le malheur en direction de B, C et D, selon l'expression éthiquement si juste de mon ami Martin Andler).

Mais j'aurais le sentiment de ne pas prendre au sérieux la situation, de ne pas m'engager véritablement dans l'hommage, si je n'en venais pas maintenant à lire et discuter Francis Wolff. Je le ferai, je l'avoue, sur la seule base de ses deux ouvrages Dire le monde et Notre humanité, des contributions de «philosophie générale » qui m'ont semblé a priori (peut-être à tort) les mieux proportionnées à mon éventuelle réaction.

\section{Dire le monde}

Au sujet de ce premier ouvrage, je dois commencer par exprimer une surprise, en résonance avec ce que j'ai déjà dit dans mon préambule. Ma surprise fut, en lisant cet opus, de découvrir que le point de vue, pour l'essentiel, était transcendantal. Je le dis au sens suivant: Wolff s'attache à décrire les structures que le monde ne peut éviter d'endosser si nous devons pouvoir le dire et l'accueillir dans un langage nôtre. Cela revient à déployer, au niveau langagier, une épure transcendantale de toute réalité : à expliciter les traits que porte et affiche nécessairement le réel s'il doit arborer l'uniforme de réel connu dans et par notre langage. On reconnaît dans de telles formules un décalque de celles qui décrivent les structures a priori de la réalité selon Kant : à ceci près que, chez lui, le crible par lequel passe la réflexion transcendantale en vue de leur dégagement n'est pas le même, il est celui de la phénoménalité et de la discursivité conjuguées, et non pas celui du langage et de la proposition.

Or, j'anticipais plutôt chez lui, voyant Wolff comme un champion du renouveau de la métaphysique, une énonciation abrupte des structures ultimes de la réalité telles qu'en elles-mêmes, de l' «absolu », comme on l'appelle en mode continental. Pourtant, non seulement il n'en va pas ainsi, mais Wolff amorce sa méditation par la description des divers « milieux totalitaires » qui nous piègent, au sens où nous ne pouvons pas vraiment leur échapper ni en sortir, s’il est vrai que nos façons de tenter de le faire peuvent être revendiquées par le milieu en question, réduisant et ruinant notre échappée putative. Il mentionne ainsi la conscience aussi bien que le langage, et l'on comprend, du coup, que le transcendantal puisse être prononcé au moins deux fois, une fois de façon 
phénoménologique, et une fois de façon logico-linguistique. Ce qui serait, donc, en substance ce que fait Wolff dans son livre.

Pour rester encore un instant sur ce point, Wolff fait un petit peu plus que je n'ai dit, va un peu plus loin : il constate que, à certains égards, l'énonciation du transcendantal et celle de la vérité métaphysique sont indiscernables. Lorsque nous déclarons des structures de l'être qui sont déduites de son destin de dictum - de ce seul fait qu'il est appelé à se montrer comme ce que nous dépeignons dans nos énoncés - nous pouvons les déclarer dans la relativisation à nous/notre langage, ou bien, prenant acte, avec modestie en quelque sorte, que nous ne saurions - notre convention expressive linguistique étant ce qu'elle est - concevoir qu'il en soit autrement, déclarer les mêmes structures absolument : il n'y a pas place en nous pour ne pas «valider » la structure transcendantale.

Je pense que la possibilité d'une telle ambiguïté ou d'un tel recouvrement est constitutive de l'espace ouvert par Frege : un espace qui, nous sommant de reconnaître que nous ne sortons jamais de la revendication de la référence, supprime l'écart propre à la posture épistémologique, et permet que l'on reverse le transcendantal dans la positivité. Cependant, même s'il mentionne cette double valeur indifférente, Wolff ne la prend pas vraiment pour son compte comme indifférente, et il maintient tout au long de son livre la position kantienne d'un en soi inconnaissable « au-delà » de la structure qu'il décrit : du moins est-ce ainsi que je l'ai lu et compris.

Cette première observation une fois faite - elle est appelée à revenir dans mon commentaire - il me faut maintenant parler de la première grande «mise au point» qu'apporte Dire le monde. Il s'agit, on le sait, d'une mise en perspective de notre langage usuel sur le monde comme une sorte d'intermédiaire ou de compromis entre deux langages-limite, celui des choses et celui des événements.

En fait, Wolff commence par dégager une situation fondamentale, en liaison avec la figure inquiétante d'Antisthène. Ce dernier, on le sait, soutenait que l'on ne pouvait pas se contredire, puisque, quand il semblait que cela arrivait, ce qui se produisait en vérité était que chacun parlait de la chose qu'il avait en vue, et que les deux choses en question n'étaient pas les mêmes. La morale, pour Wolff, c'est qu'un langage conséquent sur le monde doit permettre d'une part que nous parlions des mêmes choses, d'autre part que nous n'en disions pas la même chose, notamment que nous puissions nous opposer, un interlocuteur attribuant à telle chose une propriété, l'autre la niant d'elle. Un des 
éléments qui «sort» de cette analyse dérivée d'Antisthène, c'est la structure fondamentale de la déclaration : $P(a)$, une propriété $P$ est dite d'un individu $a$. Dans les diverses interventions de Wolff qu'il m'a été donné d'entendre, il met toujours en avant cette forme comme la forme fondamentale de la raison et du langage à la fois. Je ne peux m'empêcher, à chaque fois, de ressentir à cet endroit la gêne d'un anachronisme. La révolution frégéenne n'est-elle pas venue, en effet, amender cette image, en nous imposant d'une part de prendre pour structure fondamentale de la prédication la forme $R\left(t_{1}, \ldots t_{\mathrm{n}}\right)$, qui ne préjuge pas du caractère unaire de l'assertion relationnelle, en nous forçant d'autre part à reconnaître comme forme la plus générale celle de la formule du calcul des prédicats du premier ordre quelconque, incluant comme ingrédients les connecteurs logiques et les quantificateurs ? En même temps, je suis plein de doutes à l'égard de ma perception d'anachronisme. Le discours de Wolff, qui reprend au fond la forme $S$ est $P$ discutée dans Kant et dans Hegel [sauf que $S$ est une fois pour toutes un nom d'individu], ne pourrait-il pas être suffisant pour ce qu'il a en vue ? Quelle différence la prise en compte de la quantification, par exemple, serait-elle susceptible d'apporter ici ?

Pour en juger, il faut peut-être passer, justement, aux deux langages-limite : le premier, le langage des choses, se contente au fond de nommer les choses, chacune ayant son nom exclusif et personnel. Toute la fonction du langage s'épuise alors dans cette « reconnaissance » de l'individualité des choses, il n'y a rien à dire de chacune une fois qu'on l'a nommée. C'est comme si la nomination en avait cerné l'essence ou le contenu d'un seul coup. Il en résulte notamment que le langage ne saurait former de proposition.

L'alternative pour Wolff, est un langage des événements, un langage qui ne se tourne vers aucun individu stable, mais se contente de déclarer que des événements $P$ surviennent. Le $P$ de $P(a)$ est gardé, mais comme événement : il n'est plus propriété, puisqu'il ne s'attribue à rien. La déclaration occurrentielle $P$ ne saurait être une proposition, pas plus que la nomination $a$ ne l'était. L'idée wolffienne est bien que notre langage est un langage d'articulation de contenu précisément parce qu'il n'est aucun de ces deux langages limite, mais plutôt leur conjonction ou combinaison judicieuse. Et cette conjonction, c'est, « originairement », la forme $P(a)$ qui l'accomplit : ce que je repérais comme anachronisme apparaît à l'examen comme une thèse sur la «logique du langage ». On en tirerait que la prise en compte des relations $n$-aires et de la quantification introduisent seulement des strates de complication supplémentaires. 
Dans le livre de Wolff survient à vrai dire, en rapport avec la détection de notre « forme fondamentale », une assez longue méditation sur des conceptions impossibles qui nous viennent nécessairement à l'esprit : celle d'un événement qui serait l'événement origine cause de tous les autres, celle d'une substance fondamentale qui envelopperait toutes les autres, ou dont toutes les autres seraient des accidents. Pour Wolff, un tel événement privilégié serait à la fois une substance nommable, et une telle substance privilégiée serait à la fois un événement ${ }^{1}$. C'est notamment en cela que ces conceptions ne tiennent pas, si j'ai compris. Mon sentiment de lecteur, à cet endroit, est que Wolff fait ici intervenir implicitement la métaphysique ancienne dont il est grand connaisseur. Pour lui, ce que je désigne dans mon langage, les référents répondant aux constantes individuelles du type $a$, sont des substances en même temps, et la quiddité de ces substances. Le langage de la nomination est également un langage de l'essence. Pour un regard contemporain et dépourvu de culture comme le mien, cela ne va pas de soi. Le langage limite de la nomination ne vaut pas immédiatement comme langage de l'essence et de la substance à mes yeux. De même, je résiste à l'interprétation de la prédication comme événement. L'argument implicite, je pense, est que s'il n'y a pas d'individus, alors au moins, il faut qu'il y ait des événements : autant dire qu'on se place au plan d'une ontologie et des concepts qu'elle peut ou doit se donner pour avoir quelque vraisemblance. Mais pour le regard contemporain dépourvu de culture, un prédicat du langage n'est pas déjà un événement: au contraire, envisager les prédicats comme événements, c'est une façon de mettre en délicatesse la forme obligée de déclaration du monde $R\left(t_{1}, \ldots, t_{n}\right)$. En revanche, la lecture wolffienne rejoint celle de Deleuze, il est vrai inspirée selon lui des stoïciens : la proposition exprime un événement, ce qui veut notamment dire pour lui que le prédicat enveloppé dans la proposition, en tant que véhicule ou effecteur de sens, doit être regardé comme événement : le noème du vert de l'arbre, dit-il en reprenant Husserl, demande à être compris comme le verdoyer de l'arbre.

Mon sentiment est le suivant : pour donner consistance aux « langages-limite », à l'un comme à l'autre, il faut sortir de la logique, et convoquer des raisons et des notions

\footnotetext{
${ }^{1}$ Wolff analyse les concepts métaphysiques de substance et de fondement comme, respectivement, celui de Ur-chose qui enveloppe tout événement, et celui d'Ur-événement pris comme chose. Il signale aussi que Dieu a toujours été considéré comme les deux à la fois, donnant support à une tension irréductible.
} 
ontologico-philosophiques « pré-logiques », comme la philosophie grecque peut nous en donner, mais aussi certaines philosophies contemporaines que Wolff a fréquentées.

Mais finalement, ce qui importe à Wolff, c'est de comprendre au niveau de la forme de notre dire le monde la possibilité des actions, c'est-à-dire de propositions exprimant des actions. Son problème ressemble à celui, qu'il évoque, de Davidson dans Essais sur l'action et l'événement, lorsque ce dernier cherche à caractériser les actions parmi les événements. Il ressemble aussi, et finalement, comme je vais essayer de le justifier, ce fil me paraît plus significatif, à Lyotard allant chercher - dans Le différend - une philosophie du langage frégéano-austino-wittgensteinienne pour présenter le monde de notre pratique, notre éthique et notre politique.

D'une façon qu'on peut juger surprenante, Wolff ne se satisfait pas d'une pragmatique ordinaire, insistant par exemple sur la dimension illocutionnaire, pour penser l'action : pour lui, le marqueur grammatical Je du sujet de l'énonciation est la chose essentielle. Le rapport de l'énoncé avec cette instance, à laquelle se réfèrent tous les indexicaux, fait de cet énoncé une parole, de l'événement qu'elle dit une action, et elle soustrait l'auteur et l'événement de son action à la pure logique en troisième personne, conduisant nécessairement à traiter l'agent comme une substance nommable et l'action comme un événement causalement dérivable. Lorsqu'on essaie de penser dans les termes du « langage du monde », on ne peut faire mieux que d'imputer l'action à une entité-limite, la personne, qui serait à la fois chose et puissance d'événements ultime, ce qui n'est pas tenable ( $c f$. ce qui était dit sur la substance). En revanche, de la prise en compte du Je découle finalement un «monde de la parole » qui n'est pas le même que le monde corrélat d'un langage attribuant des propriétés à des individus évoqué à l'instant. Wolff insiste beaucoup sur ceci que le fait originaire, en l'occurrence, est que des événements, via la parole, deviennent des actions «miennes » : la mienneté des actions rend possible l'évaluation, l'axiologie, l'imputation.

Si l'on veut, Wolff retrouve, par son analyse linguistique et son recours à la dimension "pragmatique" - comprise par lui dans les termes du $J e$ - la distinction kantienne entre la vue déterministe en troisième personne sur nos actions et leur vue « responsabilisante » dans les termes de la causalité intelligible révélée par l'impératif catégorique. En vérité, il fait plus : il justifie et redéploie la figure du «sujet originairement synthétique », en montrant comment le Je de l'indication et de la parole est présupposé dans toute identification d'objet. 
Comme je le suggérais un peu plus haut, la démarche de Wolff me rappelle, à certains égards, celle de Lyotard dans Le différend. En un sens, Lyotard, lui aussi, veut partir de la donne d'un monde qui est ce que dit notre langage, pour arriver à une pensée (nouvelle) de l'affaire éthico-politique. Deux différences pourraient ici être marquées : 1) D'abord, Lyotard se donne d'emblée la dimension pragmatique, en décrivant la «présentation d'univers» accomplie par chaque phrase comme consistant en l'introduction d'un destinateur, d'un destinataire, d'un sens et d'un référent ; il superpose en quelque sorte l'enseignement de Frege et celui d'Austin. À une telle décision correspond, sans doute, le fait que le monde mis en scène à travers «l'ontologie des phrases » est d'emblée le monde de l'intersubjectivité (bien qu'il soit aussi le monde de l'objectivité pour Lyotard, pour ainsi dire à l'arrière-plan). 2) L'orientation de Lyotard est d'aller jusqu'au politique, sa grande affaire. Il traverse l'enjeu éthique, à travers son commentaire de Kant et de Levinas essentiellement, mais il ne s'agit que d'une étape. À l'inverse, il semble en lisant Wolff que la question de la possibilisation de l'éthique soit le plus important pour lui. Dans cet ouvrage, la perspective ou la dimension du politique ne sont pour ainsi dire pas abordées.

Poursuivant ma réflexion limitée sur le travail de Francis Wolff, je m'intéresse maintenant à son ouvrage Notre humanité. Je sais gré, pour commencer, à notre auteur d'avoir reconnu la question de l'humain - c'est-à-dire encore de ce que nous concevons comme l'essence ou le trait déterminant de cette humanité que nous supposons notre appartenance - comme centrale et décisive pour le débat contemporain. Poser une telle question, c'est une des manières d'échapper à l'éviction péjorative de l'enjeu subjectif dans la philosophie française des années 60-70. Une éviction qui fut la règle, et qui domina toute expression philosophique. Qui de plus, curieusement, n'a pas été abolie, y compris dans les pensées de ceux qui ont voulu s'écarter de ce style. Les adeptes des paradigmes phénoménologiques et analytiques - les deux voies méthodologiques alternatives à cette non-méthodologie que fut la pensée française des années 60-70convergent pour l'essentiel avec la volonté de surmonter toute pensée en termes du sujet : les uns placent le monde et ses aspects, les affects qu'il commande même, avant l'instance qui éprouve, prônant une phénoménologie non subjective et non transcendantale; les autres placent le langage et l'architecture logico-compositionnelle de ce qu'il permet d'échanger avant celui qui émet les propositions. 
Se demander ce qu'il en est de l'humain, c'est se demander, malgré tout, quelle est la retombée pour nous ou auprès de nous de ce que nous pensons, de notre méthodologie elle-même éventuellement. C'est remarquer qu'aucune décision ou dévalorisation, aucune critique ardente du narcissisme, ne peuvent retirer à une telle question sa pertinence et son importance. «Pour nous» certes, mais quelle autre finalité est opposable à celle du pour nous, pour l'entreprise « humaine, trop humaine » de la philosophie?

Le livre de Francis Wolff, cela dit, nous propose quatre figures de l'humain, dont la présentation occupe la part prépondérante du livre. Ces quatre figures sont celles de l'animal rationnel d'Aristote, de l'esprit (ineffablement) uni au corps de Descartes, celle de l'homme structural défendue par les penseurs des années 60-70, et celle de l'homme neuronal, donnant son titre à un livre de Jean-Pierre Changeux, mais compris par l'auteur comme à l'arrière-plan de beaucoup de démarches contemporaines.

Francis Wolff considère, au fond, chacune de ces figures comme toujours vivante, comme une ressource pour envisager l'humain ici et maintenant, même si seule la dernière appartient au présent contexte épistémologique et philosophique. Chacune de ces figures, de plus, est pour lui à la fois une figure métaphysique, une figure épistémologique et une figure éthico-politique. A partir d'un placement de l'humain dans l'ordre général de l'être, se détermine selon lui une conception de la connaissance possible et une prescription de ce qui est souhaitable, obligatoire ou interdit dans l'ordre pratique.

Il faut ici, avant toute chose, faire l'éloge de la clarté et de la force avec lesquelles l'auteur présente chacune de ces figures. Tout à la fois, mettant en œuvre un esprit de synthèse admirable, il la ramène au noyau conceptuel de ce qui importe le plus, et il signale les difficultés ou les interprétations erronées, dans un travail de mise au point redressant les préjugés particulièrement appréciable.

Je voudrais, cela dit, signaler à cette occasion ce qui est pour moi un symptôme, revenant sur un point déjà évoqué, correspondant à une difficulté de réception que j'ai et qui tend à se maintenir, même si je comprends que je ne dois pas m'en tenir à mes premières réactions. Pour moi - peut-être précisément parce que j'ai été, à l'origine, l'élève de Lyotard - il est de prime abord inacceptable que le métaphysique commande l'épistémologique et le pratique. C'est pourtant justement pour ce motif, je pense, que le métaphysique connaît une telle faveur, et se voit compris comme la part la plus haute de 
la recherche philosophique par beaucoup. Or, resté kantien à cet égard, il me semble insurmontable que les modes de légitimation dans le domaine ontologique, dans le domaine épistémologique et dans le domaine pratique ne sont pas les mêmes, et que les acquis dans une entreprise ne sont pas eo ispso des acquis dans les deux autres. La démarcation entre la diction ontologique et la diction épistémologique correspond chez Kant exactement au rejet de la métaphysique dogmatique. Kant donne la priorité à la question transcendantale de la possibilité de la connaissance, et il en conclut que la connaissance universelle et générale de l'absolu, ne reposant sur aucun autre élément que le caractère incontournable de l'accord de l'entendement avec lui-même, se limite à l'épellation des formules universellement valides de la logique (pour l'exprimer de façon contemporaine), c'est-à-dire est vide ou triviale ${ }^{2}$.

De cet écart qui demeure, je discerne un symptôme dans le livre Notre humanité. La seconde figure de l'homme pour Wolff est clairement celle de l'homme « moderne » après l'homme « antique » (si l'on me passe l'expression un peu bizarre), un homme qui se voit explicitement associé, dans l'ordre de la connaissance, à l'essor de la physique mathématique. Chez Wolff, la figure de l'humain sous-jacente est la figure cartésienne de « l'union de l'âme et du corps ». J'avoue que je tique ou que je sursaute à cet endroit. À mon gré, c'est Kant qui devrait ici intervenir, avec sa philosophie transcendantale. Certes, Descartes a pensé, lui aussi, que la mathématique avait tout pouvoir pour modéliser le réel, mais il l'a pensé, justement, de façon métaphysique, ajoutant crédit - dût-ce être, en dernière analyse, par le truchement d'une interprétation de Dieu - à la constitution ultimement mathématique de l'être mondain (comme le fait Leibniz, dans un langage autre mais cousin). Or, pour ce que décrit Wolff, il me semble important que l'on s'en tienne à la formulation kantienne : pour le maître de Königsberg, la mathématicité de toute science authentique résulte de ce que la mathématique est le lieu de l'a priori et que la science coïncide avec le projet d'une connaissance universelle a priori. La physique mathématique est donc l'orientation souveraine que nous connaissons par notre faute et de notre fait, plutôt qu'elle résulterait d'une assurance métaphysique quelconque. Mais la figure corrélative du sujet n'est, du même coup, pas la même. Chez Descartes on a l'union ineffable de l'âme et du corps, ainsi que le livre de Wolff l'expose et l'explique

\footnotetext{
${ }^{2}$ Sur ce dernier point on peut éventuellement songer à le critiquer, en suivant Hintikka par exemple.
} 
lumineusement. Chez Kant en revanche, on a la dualité du sujet empirique factuel et d'un sujet de la science identifié comme sujet transcendantal, qui n'est pas une structure réelle : plutôt une structure juridique, un ensemble de droits encadrant des pouvoirs relatifs au jugement et à l'intuition.

Mon sentiment est que cette divergence que je marque, correspondant à la question du traitement de la figure «moderne », retentit sur les deux figures venant ensuite : il n'est nullement indifférent que l'homme structural et l'homme neuronal (ou l'homme cognitif? ou l'homme naturalisé ?) se soient volontiers fait valoir en liaison avec un rejet de la philosophie transcendantale, une réfutation de celle-ci. Et il est intéressant pour nous de comprendre que l'homme structural et l'homme neuronal ont eu d'autant plus de mal à assurer leur posture que l'ennemi qu'ils se donnaient ne parlait pas le même langage, et introduisait la couche du transcendantal au-delà des positivités.

Un dernier point général m'intéresse, après la lecture de Notre humanité. Le morceau de bravoure de la fin du livre est la discussion par Francis Wolff des « conséquences » pratiques tirées des diverses figures métaphysiques de l'humain. À l'intérieur même de ce morceau de bravoure, la vedette est tenue, il me semble, par son débat avec certaines orientations éthiques prétendument déduites de la thèse métaphysique posant l'homme comme « un animal comme les autres », notamment l'orientation dite « animaliste ».

Une fois de plus, je commencerai par formuler une réticence, qui correspond toujours au même écart : me tenant dans la filiation - cette fois plus bigarrée que dans les discussions précédentes - de Hume, Kant, Moore, Wittgenstein, Levinas et Lyotard, je résiste à l'idée que l'on puisse dériver un prescriptif d'un dénotatif, l'obligation de l'être, le bien pur de la description du monde, que l'on puisse traduire dans le langage naturaliste la propriété de bonté, que nos maximes pures puissent découler d'un savoir quelconque, qu'un is puisse dériver d'un ought. Donc le chapeau proposé par Wolff me gêne. Pas plus qu'une épistémologie, une morale ne peut sortir d'une métaphysique, comme je l'entends.

Maintenant lorsqu'on entre dans le détail de ce qu'énonce Wolff, dans chaque cas, on y trouve beaucoup de subtilité et de plausibilité. Je ne suis pas sûr que, si on regarde avec minutie le fonctionnement logique de son propos, il s'agisse jamais de dérivation. Wolff repère plutôt de l'extérieur des continuités entre les attitudes, qu'il ne construit pas forcément comme des déductions métaphysiques. Notamment, avant même d'entrer sur 
le terrain moral, je remarque qu'il critique la confusion, chez nombre d'auteurs ou dans nombre d'esprits, entre le principe méthodologique commandant une enquête sur l'homme, sa cognition, ses comportements, les animaux, leurs relations, ressemblances et différences, et un résultat définitif et global. Si les sciences cognitives, par exemple, doivent étudier la connaissance comme cognition, comme fait de la nature, et par suite n'ont pas à présupposer une discontinuité essentielle avec la cognition animale, cela ne signifie pas qu'elles aient obtenu de manière claire et univoque un résultat d'identité, d'homogénéité. La «rationalité cognitive» a bien souvent fait perdre de vue à ses consommateurs (qui sont parfois ses acteurs) une telle distinction. De même, beaucoup estiment que, puisque les performances cognitives de l'humain sont étudiées (ou ont été souvent étudiées) au moyen de l'analogie avec l'ordinateur (en cherchant à quel programme équivalaient nos traitements cognitifs), il est pour ainsi dire établi que notre cerveau est un ordinateur. Or il y a loin de l'un à l'autre. De ce point de vue, je trouve d'ailleurs que Francis Wolff minimise trop le débat inter-paradigmatique à l'intérieur des sciences cognitives. Même si, en dépit de l'invraisemblance scientifique du computoreprésentationnalisme, assez bien mis en lumière par les partisans du néoconnexionnisme à la fin des années 80, le paradigme symbolique est resté sociologiquement dominant, souvent en mettant de l'eau dynamique dans son vin, je pense que la question de savoir s'il ne faudrait pas traduire les recherches cognitives dans le paradigme de la modélisation continuiste qui est celui de la physique (y compris quantique, les espaces de Hilbert sont des objets incorporant $\mathbf{C}$ et donc $\mathbf{R}$ ), et la question de savoir si la description de nos performances ne doit pas se régler sur un constructivisme radical, ayant à rendre compte de l'émergence des régulations ellesmêmes, n'ont pas été tranchées et décidées de façon définitive.

Enfin, l'essentiel n'est pas là. Il réside plutôt dans la façon dont Francis Wolff, prenant au sérieux les pensées explicites et implicites des uns et des autres, montre que ni ce qui s'affichait comme connaissance scientifique de l'homme structural, ni ce qui aujourd'hui se prévaut des succès du paradigme de l'homme naturalisé, notamment neuronal, ne nous apporte ou ne nous a apporté de raisons sérieuses pour amender les principes d'un humanisme «moderne» qui reste, d'ailleurs, la référence la plus difficilement éliminable de notre droit et de nos institutions. A l'image de la faculté de clarification et d'articulation qu'il manifeste tout au long du livre, ses mises au point sur 
de tels sujets sont percutantes, et donnent le sentiment de redresser tout un ensemble de confusions usuelles.

\section{Conclusion}

Ce que je retiens comme la bonne nouvelle liée à l'œuvre de Francis Wolff, même au vu de la connaissance limitée que j'en ai prise, c'est la possibilité de maintenir et faire entendre la parole d'un rationalisme ouvert, au sein d'un espace intellectuel qui trouve mille prétextes pour s'en écarter. Certains se replient sur les avancées hexagonales d'il y a plus de quarante ans comme si elles avaient été voulues une prison. Certains embrassent la cause du nouveau rationalisme analytique sans envisager qu'il puisse envelopper luimême des biais l'empêchant d'accueillir et comprendre toutes les grandeurs de la raison. Certains se fient au naturalisme de la science comme si ses programmes de recherche étaient déjà la capture et la maîtrise des essences, et comme si, de ce qu'ils découvrent à comment nous pouvons évaluer nos affaires éthiques et politiques, la conséquence était bonne. Certains, enfin, valorisent ce qui est contemporain presque comme les dernières parutions de la culture commerciale le sont, et se montrent incapables, du coup, d'entendre ce qui a été pensé dans le passé dans sa cohérence et sa force propre.

Que le vrai rationalisme, le plus fort et le plus intelligent, soit celui qui en même temps comprend et entend les voix bigarrées de la raison des autres, c'est ce que nous rappelle et nous prouve par l'exemple Francis Wolff dans son travail. 